\title{
DNA methyltransferase expression in odontogenic cysts and tumours
}

\author{
CAROLINA CAVALIÉRI GOMES, JOÃO ARTUR RICIERI BRITO, \\ CARLOS INÁCIO ANDRADE and RICARDO SANTIAGO GOMEZ
}

\author{
Department of Oral Surgery and Pathology, School of Dentistry, Universidade Federal \\ de Minas Gerais, CEP 31270-901, Belo Horizonte, MG, Brazil \\ Received February 4, 2009; Accepted April 9, 2009
}

DOI: 10.3892/ol_00000026

\begin{abstract}
Epigenetic silencing of gene expression by promoter $\mathrm{CpG}$ island hypermethylation is promoted by the enzymes, DNA methyltransferases (DNMTs). DNMT3a is mainly involved in de novo methylation, whereas DNMT1 acts mainly as a maintenance methyltransferase. The purpose of this study was to investigate the immunoexpression of DNMT1 and DNMT3a in a set of odontogenic cysts and tumours. Formalin-fixed and paraffin-embedded tissue samples of eight radicular cysts, 10 odontogenic keratocysts (OKC), eight adenomatoid odontogenic tumours (AOT), 16 ameloblastomas and eight samples of normal mucosae were included in the study. The DNMT1 and DNMT3a proteins were identified by using a highly sensitive polymer-based system. We found that the normal oral mucosa, OKC, AOT, radicular cyst and ameloblastomas samples showed a widespread nuclear and cytoplasmic immunopositivity for DNMT1. Some radicular cysts, ameloblastomas, AOT and OKC samples presented a positive cytoplasmic reaction for DNMT3a, while negative staining was observed in the normal oral mucosa. Nuclear positivity was found only in the suprabasal cell layers of three OKC samples. Our study shows an increased expression of DNMT3a in odontogenic cysts and tumours, confirming that epigenetic mechanisms are involved in the development of these tumours.
\end{abstract}

\section{Introduction}

The term epigenetics defines the heritable changes in gene expression that are not coded in the DNA sequence. Epigenetic

Correspondence to: Professor Ricardo Santiago Gomez, Departamento de Patologia e Cirurgia, Faculdade de Odontologia, Universidade Federal de Minas Gerais, Av. Antonio Carlos 6627, CEP 31270-901, Belo Horizonte, MG, Brazil

E-mail: rsgomez@ufmg.br

Key words: methylation, odontogenic tumours, odontogenic cysts, DNMT1, DNMT3a changes are characterized mainly by DNA methylation and histone deacethylation, preventing transcription of the target gene. DNA methylation occurs when methyl groups are added to cytosine nucleotides in specific areas of the gene by the enzyme DNA methyltransferase (DNMT) (1). A number of DNMTs have been identified in mammals. DNMT3a and DNMT3b are mainly involved in de novo methylation, whereas DNMT1 acts mainly as a maintenance methyltransferase (2-4). Epigenetic silencing of the gene expression by promoter $\mathrm{CpG}$ island hypermethylation was shown to be important in the formation of a variety of cancer types including oral squamous cell carcinoma (5). DNMTs were also found to be overexpressed in tumorigenic cells (6) and in certain human tumours $(7,8)$.

Genetic alterations were already observed in certain odontogenic cysts and tumours $(9,10)$. Although a recent study showed the methylation of tumour suppressor genes in odontogenic keratocysts (OKCs) (11), the expression of DNMTs has yet to be investigated in odontogenic tumours. We investigated the expression of DNMT3a and DNMT1 in radicular cysts, $\mathrm{OKC}$, ameloblastomas and adenomatoid odontogenic tumours (AOT).

\section{Materials and methods}

Tissue samples. Formalin-fixed and paraffin-embedded tissue samples of eight radicular cysts, 10 OKC, eight AOT, 16 ameloblastomas (eight plexiform and eight follicular histologic types) and eight samples of normal mucosae were included in the study. The age, gender and location of the lesions in each group are summarized in Table I.

Immunohistochemistry. Tissue sections $(4 \mu \mathrm{m})$ were stained with DNMT1 antiserum (diluted 1:150, clone 60B1220.1, Imgenex, USA) and DNMT3a (diluted 1:100, clone 64B1446, Imgenex). Heat-induced epitope retrieval was performed with $10 \mathrm{mM}$ citrate buffer, $\mathrm{pH} 6.0$, for $30 \mathrm{~min}$ in a steamer at $96^{\circ} \mathrm{C}$. After dilution in $0.5 \%$ BSA for $18 \mathrm{~h}$ at $4^{\circ} \mathrm{C}$ primary antiserum incubation was performed, followed by conventional streptavidin peroxidase method (LSAB, Dako, Carpinteria, CA, USA). Peroxidase activity was developed with DAB (Sigma, St. Louis, MI, USA) with timed monitoring using a positive 
Table I. Clinical data of the cases included in the study.

\begin{tabular}{|c|c|c|c|c|c|}
\hline & \multirow[b]{2}{*}{ Age (mean, range) } & \multicolumn{2}{|c|}{ Gender } & \multicolumn{2}{|c|}{ Location } \\
\hline & & Female & Male & Mandible & Maxilla \\
\hline OKC $(n=10)$ & $18(06-36)$ & 05 & 05 & 06 & 04 \\
\hline $\operatorname{AOT}(n=08)$ & $24(13-53)$ & 06 & 02 & 04 & 04 \\
\hline Radicular cysts $(\mathrm{n}=08)$ & $25(14-76)$ & 03 & 05 & 04 & 04 \\
\hline Plexiform ameloblastomas $(\mathrm{n}=08)$ & $30(12-51)$ & 03 & 05 & 06 & 02 \\
\hline Follicular ameloblastomas $(\mathrm{n}=08)$ & $21(08-27)$ & 04 & 04 & 08 & 00 \\
\hline
\end{tabular}

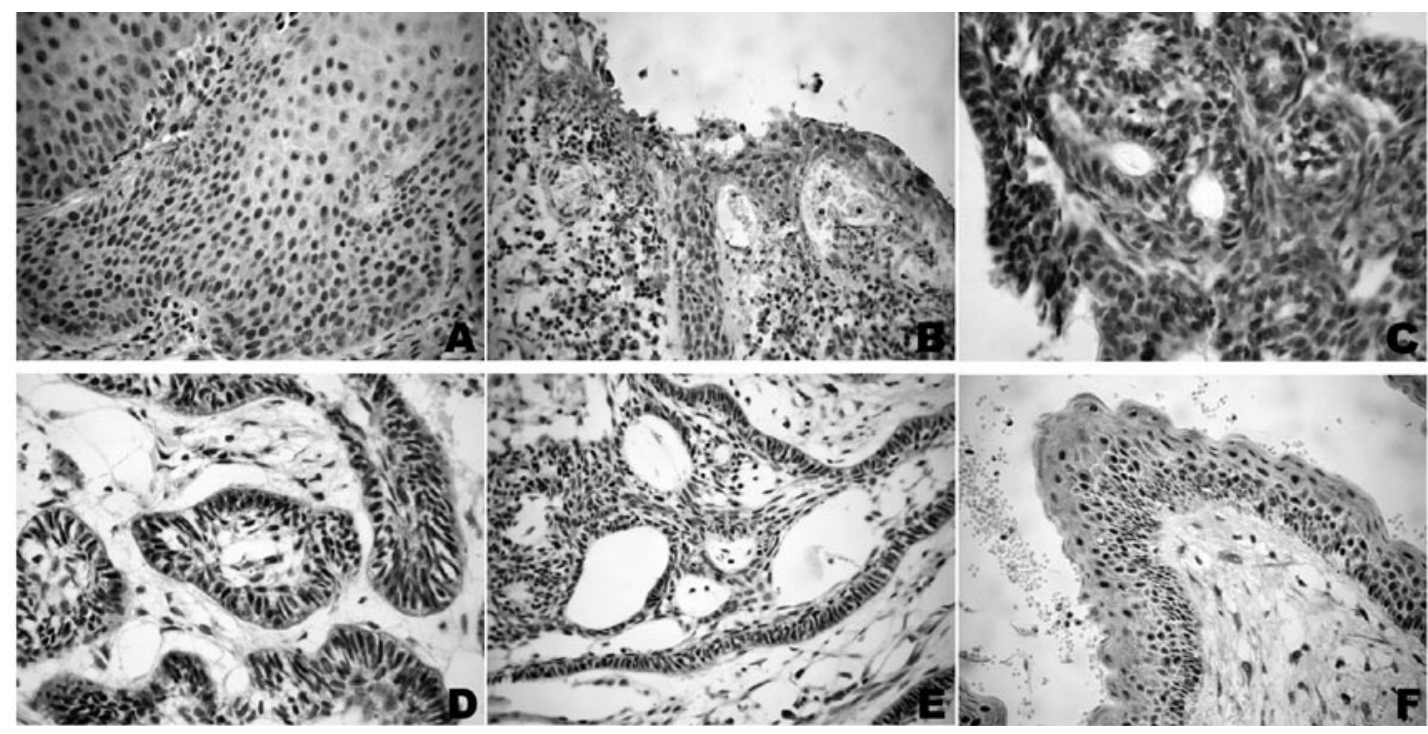

Figure 1. DNA methyltransferase 1 immunohistochemical staining in a representative case of (A) normal oral mucosa, (B) radicular cyst, (C) adenomatoid odontogenic tumour, (D) follicular ameloblastoma, (E) plexiform ameloblastoma and (F) odontogenic keratocyst. (B-F) Lesions exhibited nuclear and cytoplasmic immunostaining in all cell layers, showing the same immunohistochemical staining pattern as (A) normal oral mucosa.

control sample. For each reaction, a negative control was included. The sections were counterstained with hematoxylin.

Analysis. Only sections containing sufficient epithelium to assess the antibody reactivity were considered for this study. Two experienced independent pathologists examined multiple fields and made a descriptive analysis of each case. The samples were individually scored as Grade 1 ( $<25 \%$ of positive cells) or Grade 2 ( $>25 \%$ of positive cells). The score of the nuclear staining was performed separately from the cytoplasm.

\section{Results}

DNMT1 and DNMT3a immunopositivity was detected in the nucleus and/or cytoplasm. The OKC, AOT, radicular cyst and ameloblastoma cases showed a widespread nuclear and cytoplasmic immunopositivity for DNMT1 in all cell layers, reproducing the pattern observed in the normal oral mucosa samples (Grade 2) (Fig. 1).

All of the radicular cyst samples, 13 out of 16 ameloblastoma cases and some of the OKC and AOT cases showed a positive cytoplasmatic reaction for DNMT3a (Grade 2), but no specific pattern of positivity was observed on peripheral, central, basal or suprabasal layers (Fig. 2). Nuclear positivity for this antibody was found only in the suprabasal cell layers of three OKC samples (Grade 2) (Table II). In total, 28 (66.6\%) out of 42 odontogenic cysts and tumours showed positive nuclear and/or cytoplasmic staining for DNMT3a. The normal oral mucosa samples exhibited negative staining for DNMT3a.

\section{Discussion}

In the present report, we described the immunoexpression pattern of two catalytically active DNMTs in a group of odontogenic cysts and tumours, as well as in normal oral mucosae. To the best of our knowledge, no previous report exists about the DNMT1 and DNMT3a expression pattern in odontogenic cysts and tumours.

Hypermethylation of tumour suppressor genes that are normally unmethylated correlates with loss of expression in cancer cell lines and primary tumours. Overexpression of DNMT1 and DNMT3a was reported in human tumours (12). Previous studies a revealed significant overexpression of DNMT1 in tumour tissues $(6,13,14)$, while others found little increase of this enzyme in tumours when compared to matched normal tissue $(12,15)$. Moreover, murine DNMT1 levels were 

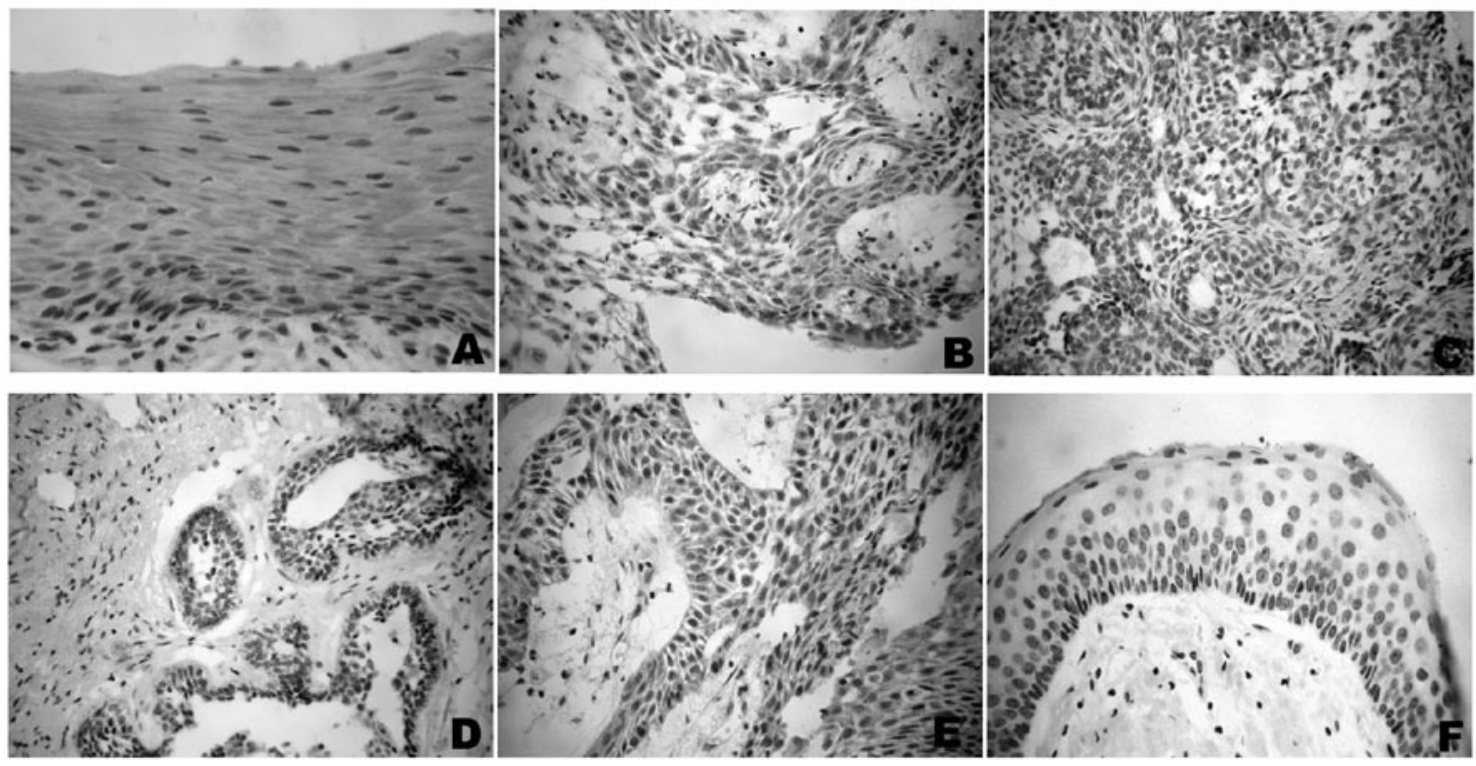

Figure 2. DNA methyltransferase 3a immunohistochemical staining in a representative case of (A) normal oral mucosa, (B) radicular cyst, (C) adenomatoid odontogenic tumour, (D) follicular ameloblastoma, (E) plexiform ameloblastoma and (F) odontogenic keratocyst. (A) Normal oral mucosa exhibit negative immunostaining, while B-E exhibited cytoplasmic staining. Note that only odontogenic keratocysts showed nuclear immunostaining and this positivity was mainly observed in the suprabasal cell layers $(\mathrm{F})$.

Table II. Immunoreactivity for DNMT3a (no., \%) in odontogenic keratocysts, adenomatoid odontogenic tumours, radicular cysts, plexiform ameloblastomas, follicular ameloblastomas and normal oral mucosae.

\begin{tabular}{|c|c|c|c|c|}
\hline Immunopositivity for DNMT3a (no., \%) & Nuclear and cytoplasmic & Nuclear & Cytoplasmic & Absence of immunoreaction \\
\hline OKC $(n=10)$ & $02(20)^{\mathrm{a}}$ & $01(10)^{\mathrm{a}}$ & $01 \quad(10.0)^{\mathrm{a}}$ & $06(60.0)$ \\
\hline $\operatorname{AOT}(\mathrm{n}=08)$ & $00 \quad(0)$ & $00 \quad(0)$ & $03(37.5)^{\mathrm{a}}$ & $05(62.5)$ \\
\hline Radicular cysts $(n=08)$ & $00 \quad(0)$ & $00 \quad(0)$ & $08(100.0)^{\mathrm{a}}$ & $(0.0)$ \\
\hline Plexiform ameloblastomas $(n=08)$ & $00 \quad(0)$ & $00 \quad(0)$ & $07(87.5)^{\mathrm{a}}$ & $01 \quad(12.5)$ \\
\hline Follicular ameloblastomas $(\mathrm{n}=08)$ & $00 \quad(0)$ & $00 \quad(0)$ & $06(75.0)^{\mathrm{a}}$ & $02(25.0)$ \\
\hline Normal oral mucosae $(\mathrm{n}=08)$ & $00 \quad(0)$ & $00 \quad(0)$ & $00 \quad(0.0)$ & $08(100.0)$ \\
\hline
\end{tabular}

aPositive staining in $>25 \%$ of cells (Grade 2). OKC, odontogenic keratocyst; AOT, adenomatoid odontogenic tumour.

shown to vary during the cell cycle and correlate with cell proliferation $(15,16)$. Although DNMT1 is considered to be a maintenance form of DNMT that copies methylation patterns after DNA replication, it has been proposed that in human cancers unknown factors target this enzyme in unmethylated substrate DNA. Therefore, some authors believe that DNMT1 protein overexpression results in de novo DNA hypermethylation during carcinogenesis (14). The cases included in our study showed a widespread nuclear and cytoplasmic immunopositivity for DNMT1 in all cell layers, as did the normal oral mucosa samples. Since DNMT1 was found in normal oral mucosa cells, it may not be relevant to the development of odontogenic cysts and tumours.

DNMT3a expression was shown to be slightly increased in tumours from bladder, colon, kidney and pancreas when compared to normal tissue $(12,15)$. We found four different expression patterns of this protein: negative, cytoplasmic, both cytoplasmic and nuclear, as well as only nuclear staining. The normal oral mucosa fragments were DNMT3a-negative. Some cases of OKC, AOT, radicular cyst and ameloblastoma showed a cytoplasmic reaction for this protein. Although the exact meaning of this cytoplasmic immunostaining is unknown, it suggests that epigenetic alterations occur in odontogenic cysts and tumours. A previous study suggested that P16 hypermethylation is involved in the malignant transformation of ameloblastoma (17), but epigenetics has been the focus of few studies regarding the pathogenesis of odontogenic tumours.

Nuclear expression of DNMT3a was observed in only three samples of OKC, mainly in the suprabasal layers. It has been demonstrated in hepatocarcinogenesis, the sequential decrease in cytoplasmic immunoreactivity for DNMT3a, as well as the concurrent increase in nuclear DNMT3a in high-grade dysplasia and carcinomas when compared to non-neoplastic and low-grade dysplasia (18). The biological significance of nuclear staining has yet to be established, but since methylation occurs inside the nuclei, we hypothesize that the nuclear expression 
of DNMT3a would mediate DNA hypermethylation events on OKC epithelial cells. Interestingly, a recent study performed by our group demonstrated the hypermethylation of some tumour suppressor genes in OKC (11).

In conclusion, our study shows an increased expression of DNMT3a in odontogenic cysts and tumours, supporting the theory that epigenetic mechanisms are relevant to the development of such lesions.

\section{Acknowledgements}

This study was supported by grants from Milênio CNPq/MCT (Conselho Nacional de Desenvolvimento Científico e Tecnológico) and FAPEMIG (Fundação de Amparo à Pesquisa do Estado de Minas Gerais), Brazil. Dr R.S. Gomez is a research fellow of CNPq.

\section{References}

1. Shaw R: The epigenetics of oral cancer. Int J Oral Maxillofac Surg 35: 101-108, 2006.

2. Bestor TH and Ingram VM: Two DNA methyltransferases from murine erythroleukemia cells: purification, sequence specificity, and mode of interaction with DNA. Proc Natl Acad Sci USA 80: 5559-5563, 1983.

3. Okano M, Xie S and Li E: Cloning and characterization of a family of novel mammalian DNA (cytosine-5) methyltransferases. Nat Genet 19: 219-220, 1998.

4. Pradhan S, Bacolla A, Wells RD and Roberts RJ: Recombinant human DNA (cytosine-5) methyltransferase. I. Expression, purification, and comparison of de novo and maintenance methylation. J Biol Chem 274: 33002-33010, 1999.

5. Ha PK and Califano JA: Promoter methylation and inactivation of tumour-suppressor genes in oral squamous-cell carcinoma. Lancet Oncol 7: 77-82, 2006.

6. Kautiainen TL and Jones PA: DNA methyltransferase levels in tumorigenic and nontumorigenic cells in culture. J Biol Chem 261: 1594-1598, 1986.

7. Sun L, Hui AM, Kanai Y, Sakamoto M and Hirohashi S: Increased DNA methyltransferase expression is associated with an early stage of human hepatocarcinogenesis. Jpn J Cancer Res 88: 1165-1170, 1997.
8. De Marzo AM, Marchi VL, Yang ES, Veeraswamy R, Lin X and Nelson WG: Abnormal regulation of DNA methyltransferase expressing during colorectal carcinogenesis. Cancer Res 59: 3855-3860, 1999.

9. Barreto DC, Gomez RS, Bale AE, Boson WL and De Marco L: PTCH gene mutations in odontogenic keratocysts. J Dent Res 79: 1418-1422, 2000.

10. Perdigão PF, Gomez RS, Pimenta FJGS and De Marco L: Ameloblastin gene (AMBN) mutations associated with epithelial odontogenic tumours. Oral Oncol 40: 841-846, 2004.

11. Moreira PR, Guimarães MM, Guimarães ALS, Diniz MG, Gomes CC, Brito JAR and Gomez RS: Methylation of P16, P21, P27, RB1 and P53 genes in odontogenic keratocysts. J Oral Pathol Med 38: 99-103, 2009.

12. Robertson KD, Uzvolgyi E, Liang G, Talmadge C, Sumegi J, Gonzales FA and Jones PA: The human DNA methyltransferases (DNMTs) 1, 3a and 3b: coordinate mRNA expression in normal tissues and overexpression in neoplasms. Nucleic Acids Res 27: 2291-2298, 1999.

13. Etoh T, Kanai Y, Ushijima S, Nakagawa T, Nakanishi Y, Sasako M, Kitano S and Hirohashi S: Increased DNA methyltransferase 1 (DNMT1) protein expression correlates significantly with poorer tumour differentiation and frequent DNA hypermethylation of multiple $\mathrm{CpG}$ islands in gastric cancers. Am J Pathol 164: 689-699, 2004.

14. Peng DF, Kanai Y, Sawada M, Ushijima S, Hiraoka N, Kosuge T and Hirohashi S: Increased DNA methyltransferase 1 (DNMT1) protein expression in precancerous conditions and ductal carcinomas of the pancreas. Cancer Sci 96: 403-408, 2005.

15. Lee PJ, Washer LL, Law DJ, Boland CR, Horon IL and Feinberg AP: Limited up-regulation of DNA methyltransferase in human colon cancer reflecting increased cell proliferation. Proc Natl Acad Sci USA 93: 10366-10370, 1996.

16. Szyf M, Bozovic V and Tanigawa G: Growth regulation of mouse DNA methyltransferase gene expression. J Biol Chem 266: 10027-10030, 1991.

17. Abiko Y, Nagayasu H, Takeshima M, Yamazaki M, Nishimura M, Kusano K, Kitajo H, Saitoh M, Kawakami T, Chiba I and Kaku T: Ameloblastic carcinoma ex ameloblastoma: report of a casepossible involvement of $\mathrm{CpG}$ island hypermethylation of the p16 gene in malignant transformation. Oral Surg Oral Med Oral Pathol Oral Radiol Endod 103: 72-76, 2007.

18. Choi MS, Shim YH, Hwa JY, Lee SK, Ro JY, Kim JS and Yu E: Expression of DNA methyltransferases in multistep hepatocarcinogenesis. Hum Pathol 34: 11-17, 2003. 